Research Article

\title{
Evaluation of Compression Performance of APM Aluminum Foam-Polymer Filled Pipes Prepared via Different Epoxy Resin Bonding Processes
}

\author{
Yanli Wang $\mathbb{D}^{1},{ }^{1}$ Qiaoyu Guo $\mathbb{D D}^{2},{ }^{2}$ Lucai Wang $\mathbb{C}^{2},{ }^{2}$ and Hong Xu ${ }^{1}{ }^{1}$ \\ ${ }^{1}$ School of Material Science and Technology, North University of China, Taiyuan, Shanxi Province 030051, China \\ ${ }^{2}$ School of Material Science and Technology, Taiyuan University of Science and Technology, Taiyuan, \\ Shanxi Province 030024, China \\ Correspondence should be addressed to Lucai Wang; wlc1985026@tyust.edu.cn
}

Received 28 March 2019; Accepted 20 June 2019; Published 15 September 2019

Academic Editor: Dora Foti

Copyright (c) 2019 Yanli Wang et al. This is an open access article distributed under the Creative Commons Attribution License, which permits unrestricted use, distribution, and reproduction in any medium, provided the original work is properly cited.

The composite structure with aluminum foam not only has the strength and toughness of the dense material but also reduces the weight of the component and increases specific deformation energy absorption performance. In this paper, advanced pore morphology (APM) foam elements are combined with thin-walled circular steel pipes by epoxy-bonding and epoxy foambonding processes to prepare composite circular pipes. The direct epoxy-bonding process using epoxy resin refers to coating the surface of APM spheres, whereas the epoxy foam-bonding process involves the mixing of the epoxy resin with the epoxy foaming agent and then coating the surface of APM spheres with this mixed epoxy resin. The compression performances and energy absorption performances were analyzed by quasistatic compression tests. Results indicate that the different bonding modes change the deformation mode of the specimen under compression. The epoxy foam-bonding APM composite pipe has a higher compression load level than the epoxy-bonding APM filled pipe. The epoxy foam-bonding APM composite pipe is superior to the epoxy-bonding APM composite and thin-wall hollow pipe. Hence, the combination of foaming and bonding of epoxy can be used as a new filling process for APM fillers.

\section{Introduction}

Reduction of vehicle weight is the key to enhance fuel efficiency, and hence different foam sandwich panels and foam-filled structures have been researched and designed for vehicles [1]. Traditional foam-filled materials and processes provide more possibilities for development of composite structures. However, in the manufacturing of large composite parts, it is difficult to precisely control the temperature factor as well as gravity drainage effects, which leads to decrease in uniformity of the porous structure of the foam core [2]. Their applications have been limited by the internal structural defects in the fabrication and difficulties associated with filling of abnormally shaped composite parts. Recently, the fabrication of composite structures using metal hollow spheres $[3,4]$ and aluminum foam elements with
APM [5-14] has become a hot topic of research. The APM element is an aluminum foam sphere with a closed-pore structure of $2-15 \mathrm{~mm}$ diameter and a complete nearlyspherical surface, which can be prepared by the modified powder-compacting foaming (PCF) method $[2,5,6]$. The geometric structure of the APM element is easy to replicate, and its uniform mechanical and physical properties are almost consistent and hence it can ensure stability of the filled structure and its performance.

This paper mainly reports the compression performance of an APM aluminum foam elements filled thin-walled stainless-steel pipe. Two different filling processes were adopted: in one process, the direct bonding used epoxy resin and the other process used the epoxy resin combined with a foaming agent. This paper studied the deformation modes under quasistatic compression. The obtained load-strain 
curve and ideal energy absorption efficiency-strain curve were analyzed and compared, which provided a theoretical basis for practical applications of the composite structure.

\section{Experimental Details}

2.1. Specimen Preparation. Figure 1 shows that the shell material used in the experiments is a thin-walled steel pipe (fabricated by Shanxi Hongxinlong Steel Trading Co., Ltd). The dimensions are as follows: outer diameter $=51 \mathrm{~mm}$, wall thickness $=0.5 \mathrm{~mm}$, and height $=80 \mathrm{~mm}$. The material is $1 \mathrm{Cr} 17 \mathrm{Mn} 6 \mathrm{Ni5N}$ stainless steel, with the following physical properties: elongation range of $55 \%$ to $60 \%$, tensile strength of $520 \mathrm{MPa}$, yield strength of $275 \mathrm{MPa}$, elastic modulus of $203 \mathrm{GPa}$, and density of $7.93 \mathrm{~g} / \mathrm{cm}^{3}$.

The spherical APM foam elements were prepared according to a procedure described as follows: the three powders, $\mathrm{Al}$ (89.2 wt.\%, technically pure, $99.9 \%, 300 \mathrm{mesh}$ ), Si (10 wt.\%, 99.5\%, 300 mesh), and a powdered blowing agent $\mathrm{ZrH}_{2}$ (0.8 wt.\%, 99.6\%, 300 mesh), were evenly mixed in a planetary ball mills with four grinding stations. The mixture was cold-pressed under $300 \mathrm{MPa}$ using a servo hydraulic press into a $\Phi 40 \mathrm{~mm}$ cylindrical preform such that the density of the preform was more than $90 \%$ of the bulk density of aluminum. Subsequently, the preform was placed in the corresponding mold and heated to $550^{\circ} \mathrm{C}$ and kept for $15 \mathrm{~min}$. Pressure below $250 \mathrm{MPa}$ was exerted by the hydraulic press, after which the cylindrical preform was transformed into a cylindrical strip with dimensions of $\Phi 10 \mathrm{~mm} \times 200 \mathrm{~mm}$, named as the foamable precursor. Then, the small granulates of $\Phi 10 \mathrm{~mm} \times 10 \mathrm{~mm}$ were cut by the wire electrical discharge machining (WEDM) process. The preform granules were transferred to a half-hollow stainlesssteel ball and placed in a full-fiber rapid-heating electric furnace at $750-800^{\circ} \mathrm{C}$ for $3-4 \mathrm{~min}$. When the preform was foamed to a near-spherical shape, it was taken out and rapidly cooled using atomizing spray. The APM foam element (Figure 2) had a nearly-spherical external morphology with a complex internal porous structure. APM elements had an average diameter of about $15 \mathrm{~mm}$, an average pore diameter of $1.879 \mathrm{~mm}$, and porosity up to $77.3 \%$ [15].

The direct bonding process using epoxy resin (paid for ChangSha BaXiongDi Adhesive Company, Ltd) refers to coating the surface of APM spheres with a $200 \mu \mathrm{m}$ thick epoxy resin layer. They were then poured into a stainlesssteel pipe and left at room temperature for $72 \mathrm{~h}$. The process of epoxy foaming of the resin involved the mixing of the epoxy resin and the foaming agent F-25 (provided by Dongguan Baibei Trading Co., Ltd) in a mass ratio of 100 : 2.5. Then, $200 \mu \mathrm{m}$ thick mixed epoxy resin coating was applied on the surface of APM spheres, which were then poured into the stainless-steel pipe. The opening of the stainless-steel pipe was sealed with a clean and smooth steel plate. After leaving it at room temperature for $72 \mathrm{~h}$, the steel plate was removed and ensured that the epoxy foaming resin was properly filled into the steel pipe.

The obtained composite pipes were all cylindrical in shape having dimensions of $\Phi 51 \mathrm{~mm} \times 80 \mathrm{~mm}$. Five specimens (Figures 3(a) and 3(b)) were prepared for each group

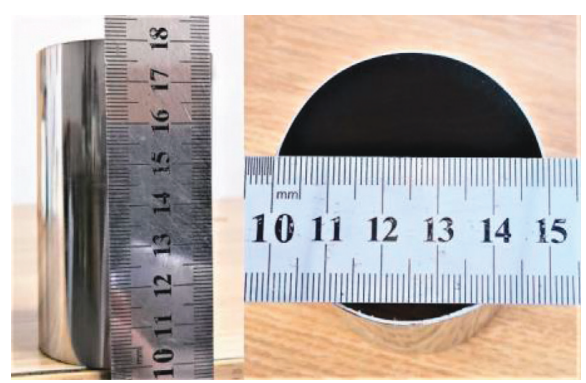

FIgURE 1: Thin-walled empty pipe.

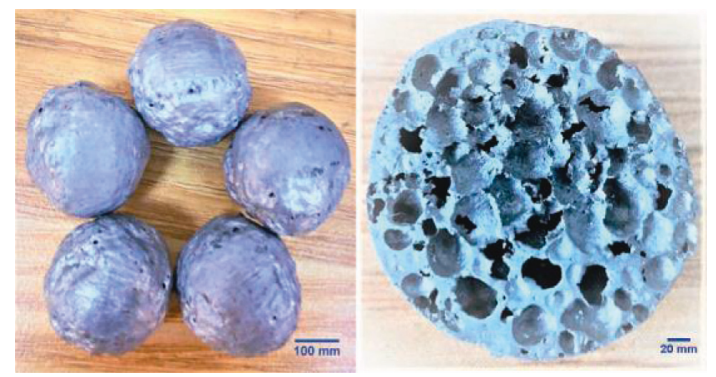

FIGURE 2: APM elements.

for carrying out the quasistatic compression test. The sample parameters are all listed in detail in Table 1.

2.2. Test Methods. The quasistatic compression test was carried out on a SANS microcomputer-controlled electronic universal testing machine (No. CMT5105). The test was carried out according to the national standard GB/T 73142017 , by the displacement loading method at a constant speed of $3 \mathrm{~mm} / \mathrm{min}$ (i.e., the initial strain rate was $10^{-2} / \mathrm{s}$ ). The applied relative displacement reached $56 \mathrm{~mm}$ (with the total strain of 0.7). Each specimen was tested under the same conditions, and the plotted load-displacement curve was processed to obtain a load-strain curve. The deformation mode of the specimen during the entire process was recorded by a camera.Energy absorption performance is one of the important indices to test a foam material. It depends mainly on the load level of the plateau on the load-strain curve and the length of the plateau. In this paper, the two parameters absorbed energy (AE) and ideal energy efficiency (Ideality) are used to evaluate the energy absorption performance of the specimen.

The equation for the calculation of the absorbed energy (AE) can be calculated by the following equation:

$$
\mathrm{AE}=\int_{0}^{d} F d \Delta,
$$

where $F$ denotes the compression force that the deformed structure was subjected to $(\mathrm{kN}) ; \Delta$ denotes the compressed length, the integration area of $\Delta$ is $(0,56)(\mathrm{mm})$; and $\mathrm{AE}$ denotes the absorbed energy ( $\mathrm{J})$.

The ideal energy absorption efficiency, $I$, denotes the ratio of the actual absorbed energy of aluminum foam to the energy absorbed by the ideal cell-type material, when both are subjected to the same strain. The ideal energy absorption 


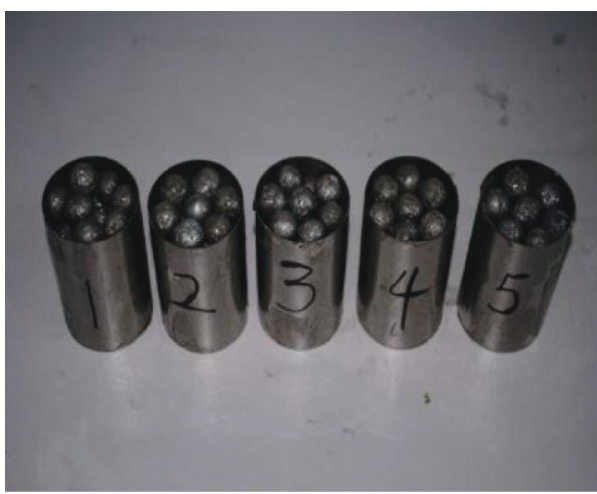

(a)

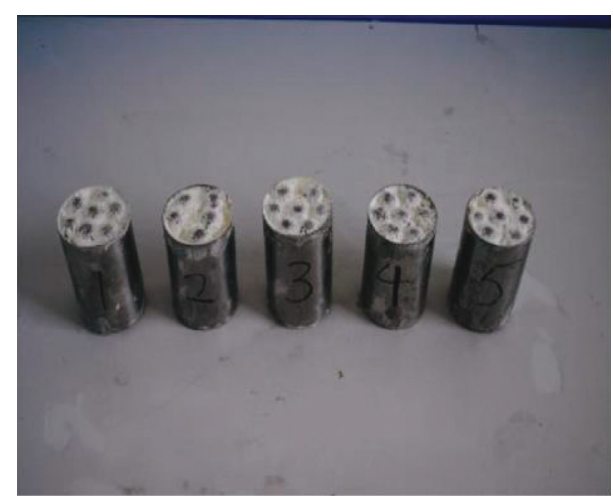

(b)

Figure 3: (a) Epoxy-bonding APM composite pipes and (b) epoxy foam-bonding APM composite pipes.

TABle 1: Parameters and physical properties of the specimens.

\begin{tabular}{|c|c|c|c|c|c|}
\hline \multicolumn{2}{|c|}{ Sample } & \multicolumn{2}{|c|}{$\begin{array}{l}\text { Epoxy-bonding APM foam filled } \\
\text { pipes }\end{array}$} & \multicolumn{2}{|c|}{$\begin{array}{l}\text { Epoxy foam-bonding APM } \\
\text { composite pipes }\end{array}$} \\
\hline Number & Diameter $(\mathrm{mm})$ & Mass (g) & Density $\left(\mathrm{g} / \mathrm{cm}^{3}\right)$ & Mass (g) & Density $\left(\mathrm{g} / \mathrm{cm}^{3}\right)$ \\
\hline $1 \#$ & 15 & 100.3 & 0.614 & 111.3 & 0.681 \\
\hline $2 \#$ & 15 & 102.6 & 0.628 & 109.3 & 0.669 \\
\hline $3 \#$ & 15 & 103.6 & 0.634 & 117.7 & 0.721 \\
\hline $4 \#$ & 15 & 100.7 & 0.616 & 108.1 & 0.662 \\
\hline $5 \#$ & 15 & 100.6 & 0.615 & 113.4 & 0.694 \\
\hline
\end{tabular}

efficiency curve is used to determine the energy absorption performance of the foam material. $I$ can be calculated by the following equation [4]:

$$
I=\frac{\int_{0}^{s} F\left(s^{\prime}\right) d s^{\prime}}{F_{\max }(s) d s},
$$

where $s$ denotes the strain, with the integral interval of $(0$, $70 \%) ; F_{\max }$ denotes the maximum load for reaching strain $s$; and the numerator denotes the curve-edged trapezoid encircled by load curve and coordinate axis within the strain interval.

\section{Results and Discussion}

3.1. Compression Deformation Mode of Pipes. Thin-walled hollow steel pipes show three deformation modes: axisymmetric (hexagonal) mode, asymmetric (diamond-shaped) mode, and hybrid mode under quasistatic loading [16]. The deformation mode of the pipe is mainly determined by the ratio of its diameter to its wall thickness, the ratio of its length to its diameter, and the material of the pipe. In general, thicker pipes undergo deformation in a hexagonal mode, whereas thinner pipes undergo deformation in the diamond-shaped mode $[16,17]$.

Figure 4 shows the results of compression on the deformation of thin-walled pipes. It obviously bends gradually in the region near the indenter, which is followed by the formation of a diamond-shaped fold. With further load, folds are continuously formed. Figure 4(b) shows the final morphology of the thin-walled pipe, after the stacking of diamond-shaped folds occurs. There are a total of four folds with larger spacings. This is consistent with the results reported in the literature [18]. According to the report [16], under quasistatic axial loading conditions, the thick-walled steel pipe (diameter-to-thickness ratio <40) mainly undergoes deformation in a hexagonal mode, while the thinwalled pipe undergoes deformation in a diamond-shaped mode. The reason for formation of first hexagonal fold was the eccentricity in the thickness of the thin-walled steel pipe.

Figure 5 shows the mode of deformation of the epoxybonding APM composite pipe. During the deformation process, obviously the fold is formed first at one end of the specimen followed by the formation of the second fold. Successively, five to six folds are formed accompanied with reduction in the spacing. Finally, the specimen exhibits deformation in a hybrid mode with diamond-shaped hexagonal folds.

It can be observed that some pipe walls sustain cracks at the folds along the loading direction (as marked in Figure 6). The fracture results due to concentration of local load as a result of interaction between the internal APM spheres and pipe wall and their co-deformation, occurring simultaneously with the formation of folds. It is evident that the deformation mode of the pipe in case of direct epoxy resin bonding is the hybrid mode, which is different from the deformation mode of the hollow steel sphere-filled thinwalled steel pipe, described in an earlier report [4]. Its sectional drawing demonstrates that the compression folds of the pipe were not completely filled.

The deformation process of epoxy foam-bonding APM composite pipe (Figure 7) shows that the bonding process of 


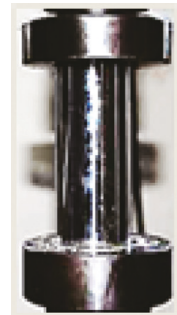

Strain $0 \%$

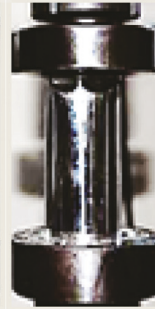

Strain $10 \%$

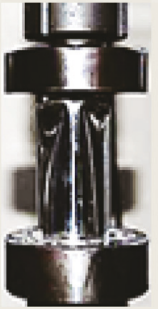

Strain $20 \%$

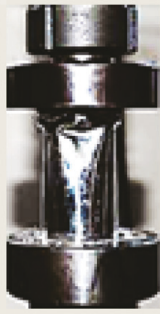

Strain $30 \%$

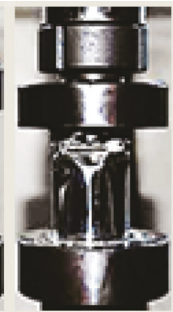

Strain $40 \%$

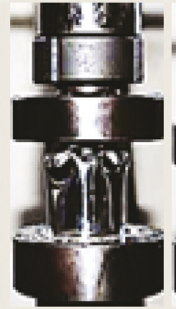

Strain 50\%

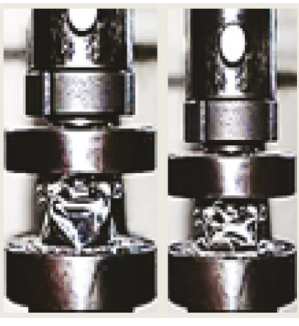

Strain $60 \% \quad$ Strain $70 \%$

(a)

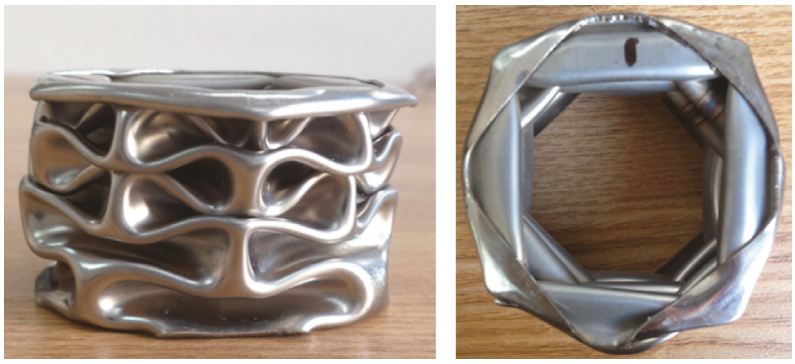

(b)

FiguRE 4: (a) Sequence showing the results of compression on the deformation of thin-walled pipes and (b) final morphology of the thinwalled pipe after compression.

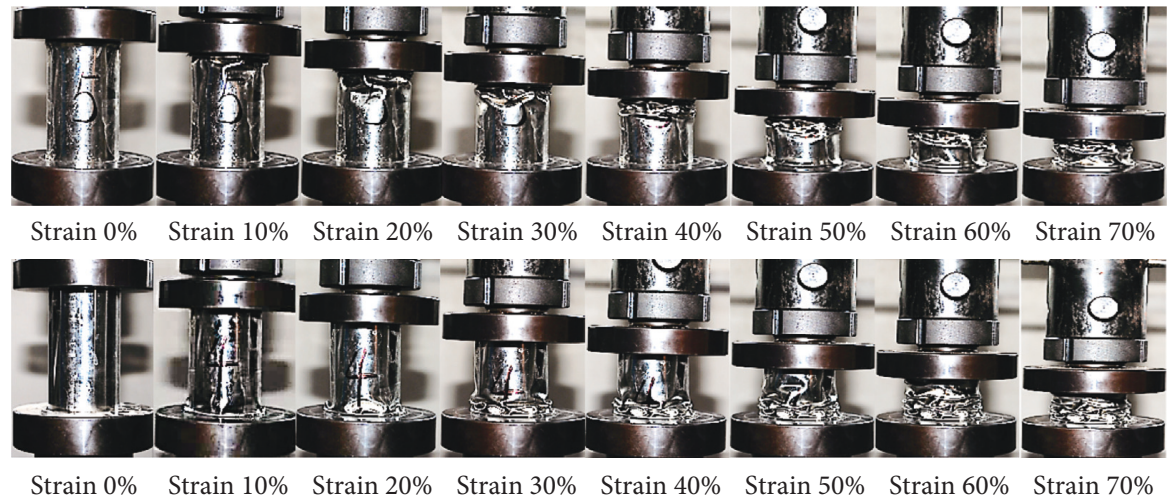

FigURE 5: Sequence showing the results of compression on the deformation of epoxy-bonding APM composite pipes.
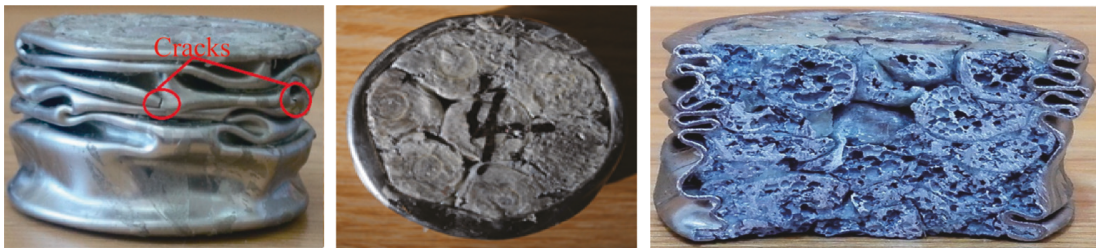

FIgURE 6: Final morphology of epoxy-bonding APM composite pipes after compression.

epoxy foam resin can effectively change the deformation mode of the filled pipe. The filled pipe deforms in a symmetrical mode, and the folds generated are all hexagonal (Figure 8). The number of folds reached seven, and the spacing between folds became smaller. The reason for this is that the epoxy resin can disperse across the pipe after foaming. When the outer surface of the aluminum alloy deforms, it collides with the aluminum foam core, which is then pressed into the folds of the outer surface and limits the inward bending of folds. Meanwhile, the inward deformation of the outer surface of steel pipe is also hindered, due to which the inward folds are not sufficiently formed. The sectional drawing shows that the pipe compression folds of the pipe have been completely filled. 


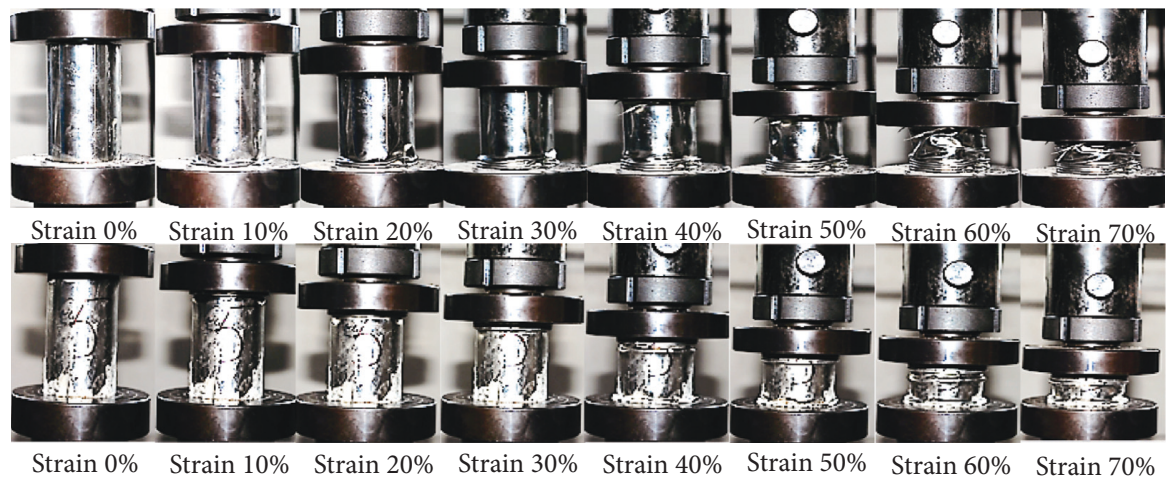

FIGURE 7: Sequence showing the results of compression on the deformation of epoxy foam-bonding APM composite pipes.
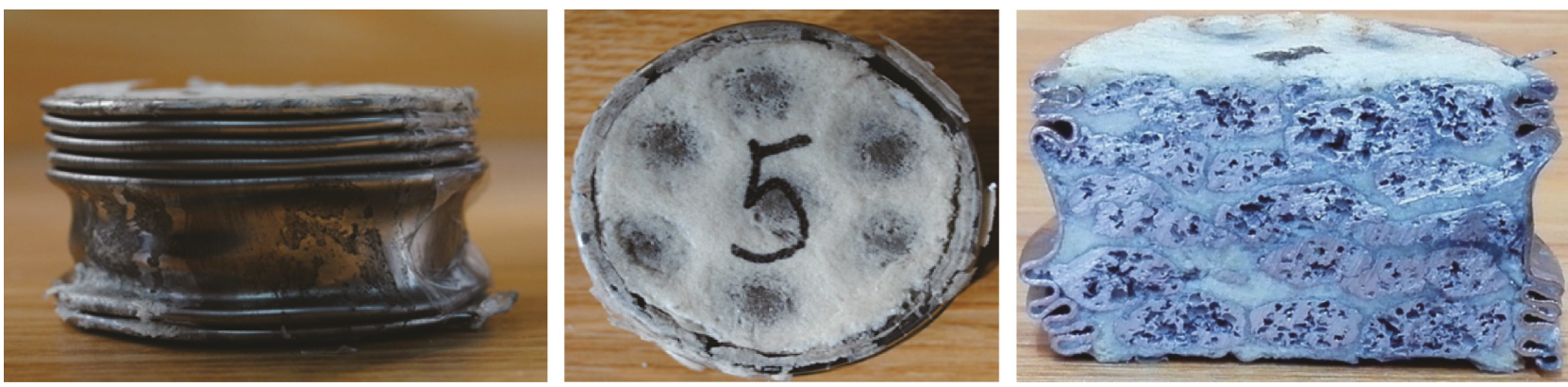

Figure 8: Final morphology of epoxy foam-bonding APM composite pipes after deformation.

3.2. Results of Compression Test. Figure 9(a) shows the loadstrain curve of the thin-walled empty pipe. After the loading begins, the load increases sharply up to its peak value and then decreases rapidly. The peak load corresponds to the initial load, which is the driving force for the formation of folds at the beginning. Initial peak load (as shown in Table 2) is useful to determine the strength of the specimen material. After the formation of the first fold, the peak value of the load decreases. As the outward folds are in mutual contact with each other, the first fold forms, then the load increases, and the second fold is again formed. The load fluctuates during the compression process. The initial peak load is higher than the sequential peak load. Additionally, the spacing between the peak load is equal to the spacing between the folds and the number of peak values is equivalent to the number of folds formed on the pipe.

Figure 10(a) shows the ideal energy absorption efficiency curve calculated from equation (2) of the thin-walled empty pipe. The ideal efficiency curve of the thin-walled steel pipe shows a sharp decrease initially and then increases rapidly. During the initial stage of the loading process, the pipe wall suffers from load and undergoes elastic deformation ceaselessly. The load increases ceaselessly but the strain remains very small, and so the process does not basically absorb energy. With further increase in the load, the pipe wall begins to yield and forms folds, and the load level decreases. At this moment, the energy absorption process begins and ideal energy absorption efficiency augments steadily. After the first fold is formed, the load increases again, and the second yield process begins, resulting in the decrease of the ideal energy absorption efficiency. The average ideal energy absorption efficiency of the thin-walled empty pipe is around $40 \%$.

Figure 9(b) shows the compressive load-strain curves of the epoxy-bonding APM composite pipe. The compression process can be divided into three stages: (1) the initial linear elastic region: the composite structure shows an elastic deformation within this region. Here, the load and strain show a nearly-linear relationship and the compressive load quickly reaches the initial peak load and then begins to yield; (2) the yield region: the compressive load begins to fluctuate and rises upwards, while the folds begin to form regularly on the pipe and the fluctuations in load are not large; (3) densification region: with further load, the small sphericalpore structure in the interior of APM pipe is crushed and forms tight aggregates. The entire specimen is almost densified, and the load increases rapidly. In comparison to that of a traditional closed-cell aluminum foam-filled pipe, the ideal energy absorption curve of the epoxy resin APM pipe shows relatively obvious fluctuations (Figure 10(b)). The pipe wall is fractured at the folds, leading to decrease in the load in curves of specimen $\# 1, \# 2$, and \#3.

Figure 9(c) shows that the load-strain curves of epoxy foam-bonding APM composite pipe are similar with those of the epoxy-bonding APM composite pipe and can also be divided into three regions: linear elastic, yield, and densification. Both curves enter the load plateau region at a strain of about $10 \%$ and later enter the densification region at a strain of about $60 \%$. The main difference lies in the strain range, where the load drop occurs. The load drops of the epoxy 

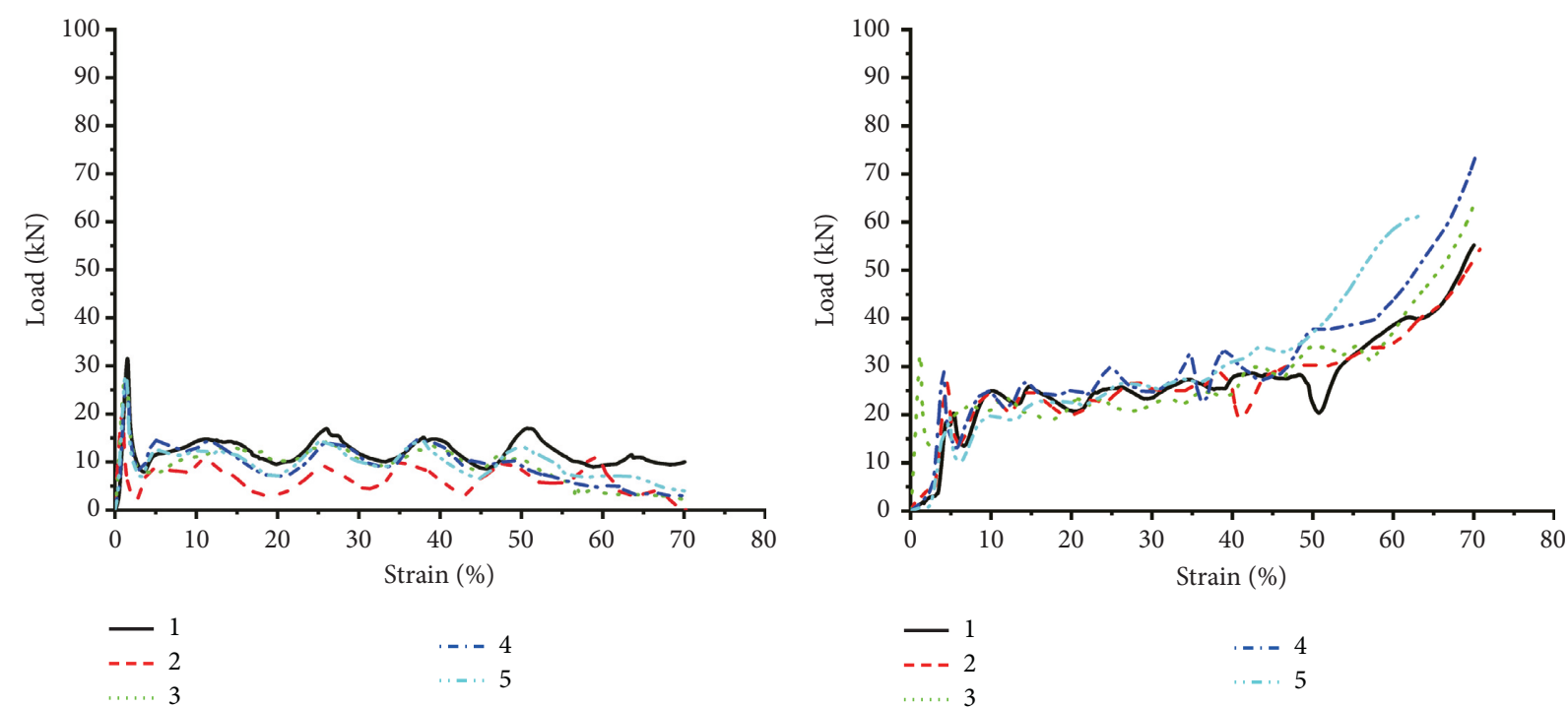

(a)

(b)

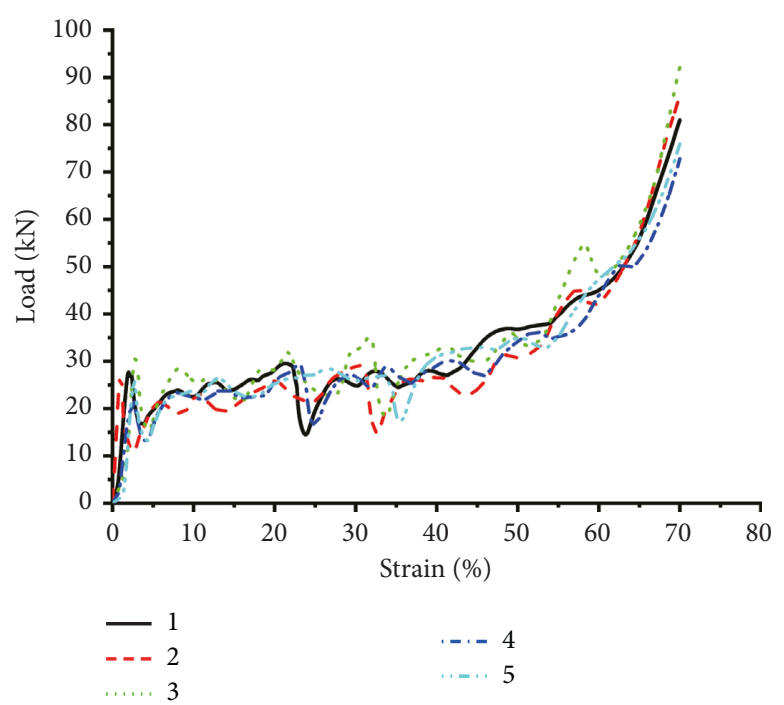

(c)

Figure 9: Load-strain curves of (a) thin-walled pipes, (b) epoxy-bonding APM composite pipes, (c) and epoxy foam-bonding APM composite pipes.

TABLE 2: Compression characteristic parameters of different specimens.

\begin{tabular}{lccc}
\hline Compression parameters & Thin-walled pipes & Epoxy-bonding APM foam filled pipes & Epoxy foam-bonding APM composite pipes \\
\hline Initial peak load (kN) & $25.57 \pm 4.26$ & $19.38 \pm 6.41$ & $22.10 \pm 7.21$ \\
Peak strain (\%) & $1.4 \pm 4 \times 10^{-6}$ & $4.1 \pm 4 \times 10^{-6}$ & $2.7 \pm 4 \times 10^{-6}$ \\
Minimum load (kN) & $8.56 \pm 1.51$ & $24.17 \pm 1.66$ & $21.74 \pm 4.53$ \\
Load drop magnitude (kN) & $14.26 \sim 19.76$ & -9.54 to -0.04 & -2.32 to 3.04 \\
Plateau load (kN) & $10.41 \pm 1.32$ & $25.99 \pm 2.32$ & $27.70 \pm 2.32$ \\
$I_{\text {average }}$ & $0.42 \pm 0.08$ & $0.70 \pm 0.07$ & $0.73 \pm 0.04$ \\
$I_{\max }$ & $0.56 \pm 0.04$ & $0.73 \pm 0.04$ & $0.74 \pm 0.06$ \\
\hline
\end{tabular}

foam-bonding APM composite pipe (-9.54 to $-0.04 \mathrm{kN})$ occur relatively earlier, approximately at a strain of $30 \%$. The obvious load drops ( -2.32 to $3.04 \mathrm{kN})$ of the epoxy-bonding pipe occur at $45 \%$ strain. The ideal energy absorption curve shown in Figure 10(c) is flatter and has a longer efficiency plateau, indicating better energy absorption performance.
3.3. Comparison of Integral Properties and Analysis. Comparison of AE values of specimens filled with different fillers in Table 3 shows that the AE of the epoxy foambonding APM composite pipe was the highest, 3.1 times more than that of the empty pipe. This was followed by the epoxy-bonding APM composite pipe, whose AE value was 


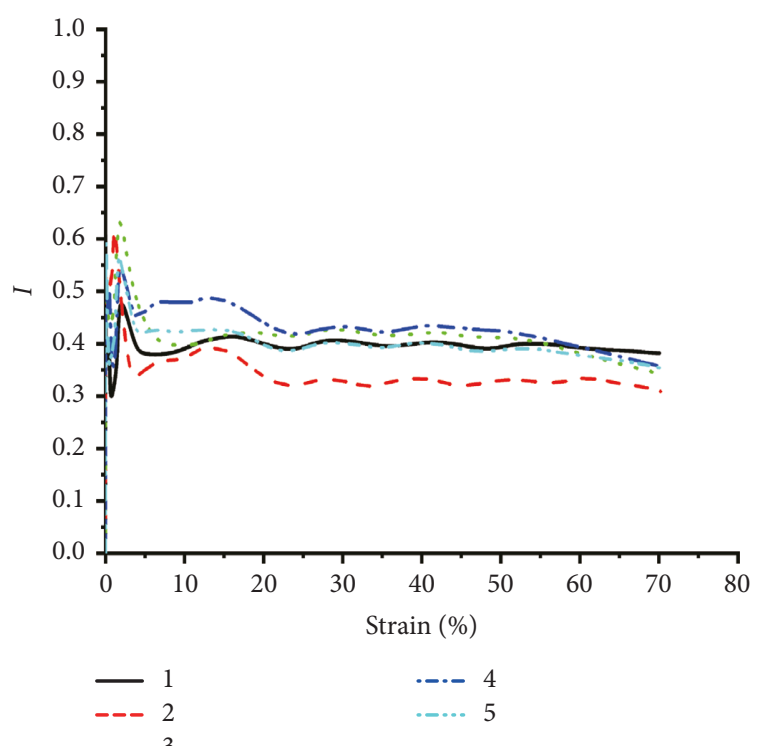

(a)

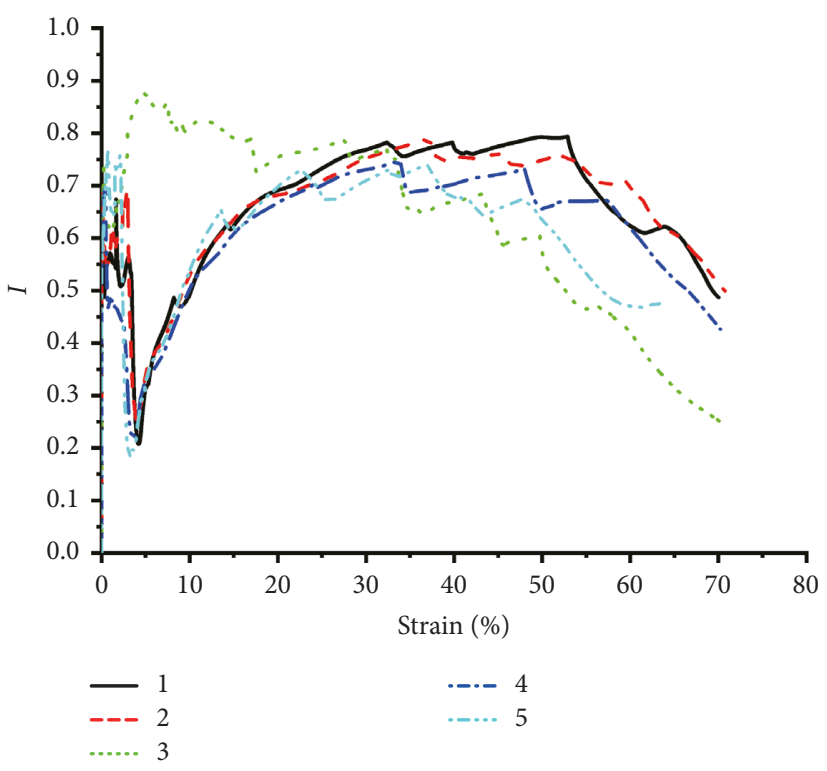

(b)

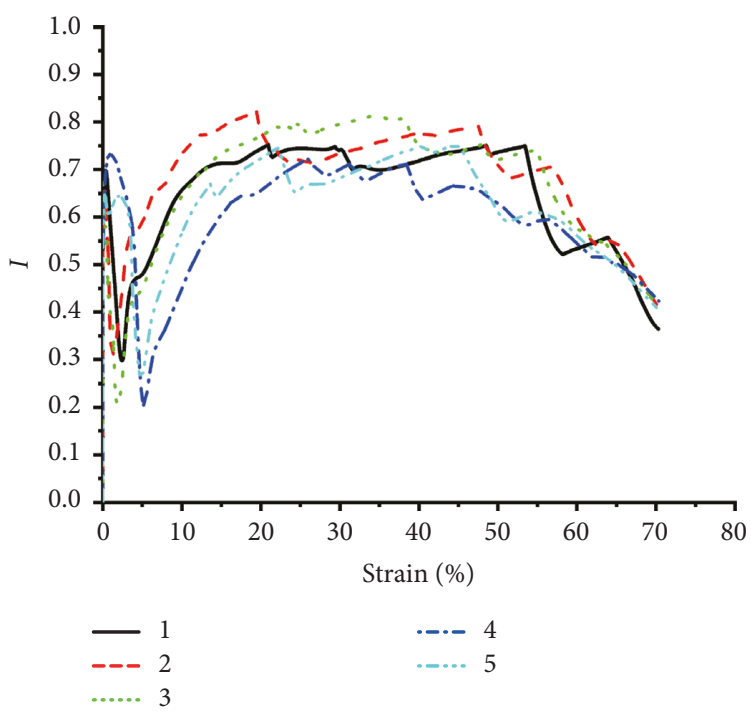

(c)

FIGURE 10: Quasistatic ideal energy absorption efficiency curves of (a) thin-walled pipes, (b) epoxy-bonding APM composite pipes, and (c) epoxy foam-bonding APM composite pipes.

2.63 times that of the empty tube. Therefore, the epoxy foambonding APM composite pipe was the best in terms of total energy absorption. The two types of composite pipe reveal the better specific energy absorption of each specimen in Table 4.

Figure 11(a) shows the load-strain curves of three pipe specimens under quasistatic compressive load. Figure 11(b) shows the average ideal energy absorption efficiency curves of the three pipe specimens. The three curves have similar trends, that is, an obvious drop in ideal energy absorption efficiency at the beginning, which corresponds to the appearance of the first peak load. At this stage, it is elastic strain. Subsequently, the yield deformation process begins, and the ideal energy absorption efficiency increases rapidly.
The plateau stage of the epoxy foam-bonding APM composite pipe starts at about $20 \%$ strain and continues until the strain reaches $50 \%$, during which the ideal energy absorption efficiency remains at about $73 \pm 4 \%$. Meanwhile, the specimen is close to densification and the ideal energy absorption efficiency reduces rapidly.

The $I_{\text {average }}$ values of the epoxy foam-bonding APM composite pipe, epoxy-bonding APM composite pipe, and thin-walled empty pipe are $0.73 \pm 0.04,0.70 \pm 0.07$, and $0.42 \pm 0.08$, respectively. Their maximum values are $0.74 \pm 0.06,0.73 \pm 0.04$, and $0.56 \pm 0.04$, respectively. The average ideal energy absorption efficiency of the epoxy foam-bonding APM composite pipe is about $154 \sim 182 \%$ that of the thin-walled empty pipe and up to $154 \sim 167 \%$ that of the epoxy-bonding APM composite pipe. From the definition of 
TABLE 3: Total energy absorption of each specimen.

\begin{tabular}{lccc}
\hline Specimen number & Thin-walled pipes, $J$ & Epoxy-bonding APM foam filled pipes, $J$ & Epoxy foam-bonding APM composite pipes, $J$ \\
\hline $1 \#$ & 674.79 & 1535.77 & 1815.72 \\
$2 \#$ & 619.82 & 1586.96 & 1711.02 \\
$3 \#$ & 525.96 & 1506.57 & 1909.36 \\
$4 \#$ & 531.80 & 1505.28 & 1702.17 \\
$5 \#$ & 552.82 & 1503.79 & 1772.68 \\
$\mathrm{AE}_{\text {average }}$ & 581.04 & 1527.67 & 1782.19 \\
\hline
\end{tabular}

TABLE 4: Specific energy absorption of each specimen.

\begin{tabular}{lccc}
\hline Specimen number & $\begin{array}{c}\text { Thin-walled pipes, } \\
J\left(\mathrm{~g} / \mathrm{cm}^{3}\right)\end{array}$ & $\begin{array}{c}\text { Epoxy-bonding APM foam filled pipes, } \\
J\left(\mathrm{~g} / \mathrm{cm}^{3}\right)\end{array}$ & $\begin{array}{c}\text { Epoxy foam-bonding APM composite pipes, } \\
\left(\mathrm{g} / \mathrm{cm}^{3}\right)\end{array}$ \\
\hline $1 \#$ & 85.09 & 2501.25 & 2666.26 \\
$2 \#$ & 78.16 & 2527.01 & 2557.58 \\
$3 \#$ & 66.33 & 2376.29 & 2648.21 \\
$4 \#$ & 67.06 & 2443.64 & 2571.25 \\
$5 \#$ & 69.71 & 2445.19 & 2554.29 \\
SAE $_{\text {average }}$ & 73.27 & 2458.43 & 2600.22 \\
\hline
\end{tabular}

Note. Specific energy absorption $=\mathrm{AE} /$ density.

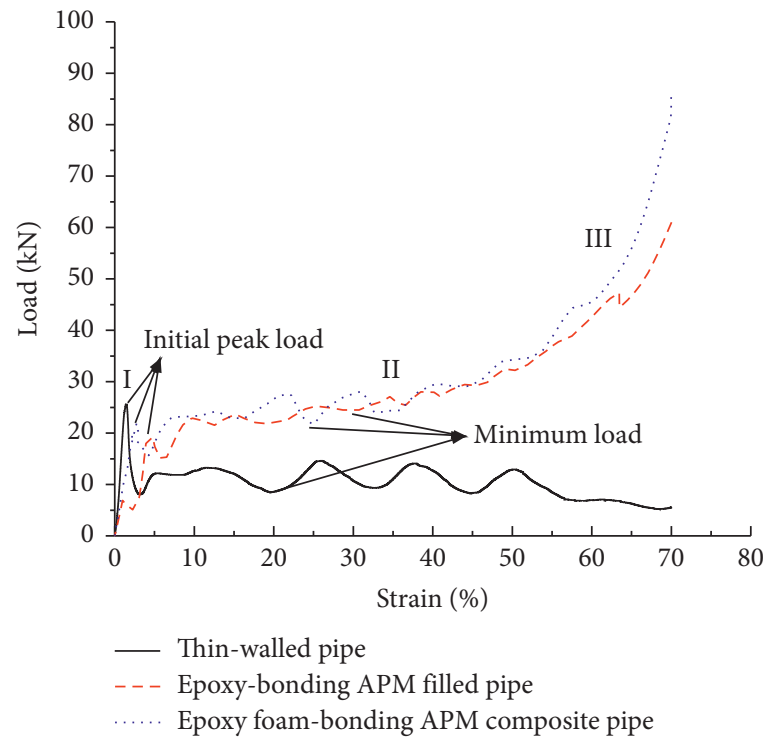

(a)

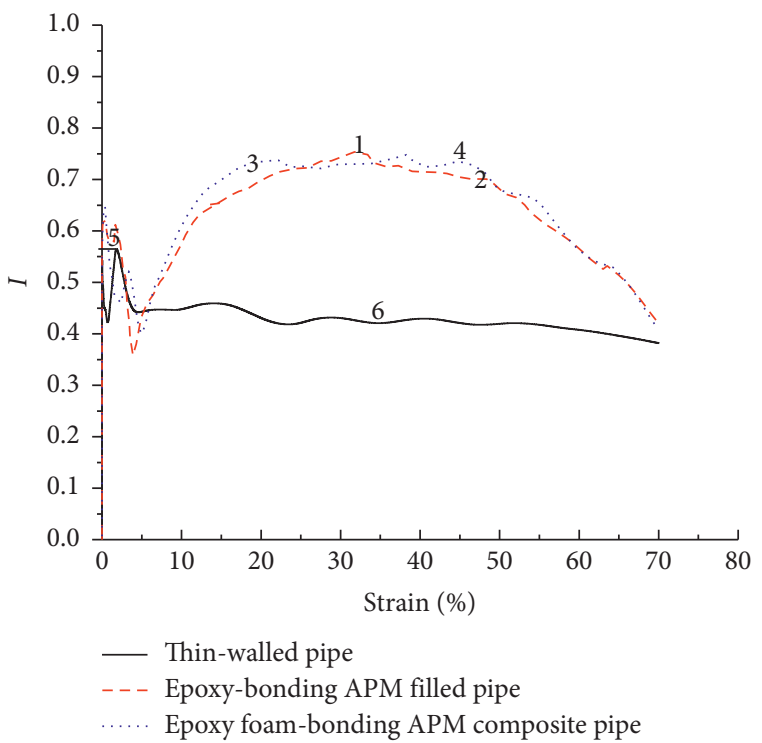

(b)

FIGURE 11: (a) Load-strain curves of three pipe specimens and (b) average quasistatic ideal energy absorption efficiency curves of three pipe specimens.

$I_{\text {average }}$ it was obvious that larger the value of actual energy absorption, the closer it is to the ideal energy-absorbing material. This indicates that the epoxy foam-bonding APM composite pipe has excellent ideal energy absorption.

Table 2 lists the parameters for compression characteristics of the three pipes. The epoxy-bonding APM composite pipe shows the first peak load at around $4 \%$ strain, while the first peak load of the thin-walled empty pipe appears at about $1.4 \%$. The difference in the position of the first peak load suggests that the formation of first fold in the epoxy-bonding APM pipe is slower than that of the thinwalled empty pipe. This also indicates that the bonding effect enhances the interaction between the APM core and the outer wall, thereby changing the deformation mode of the filled pipe. The load reduces after the formation of the fold. The average load drops of the epoxy-bonding APM composite pipe and the thin-walled empty pipe are $-10 \mathrm{kN}$ and $19 \mathrm{kN}$, respectively. The former has a smoother decrease.

At $10 \%$ strain, the epoxy foam-bonding APM composite pipe enters the high-efficiency energy absorption region and the region terminates at 55\% strain. The epoxy-bonding APM composite pipe enters the high-efficiency energy absorption region at $30 \%$ strain and remains up to about $50 \%$. The epoxy foam-bonding APM composite pipe further broadens the high-efficiency energy absorption region with ideal energy absorption efficiency over $70 \%$. 
During the preparation of the epoxy-bonding APM composite pipe, the epoxy resin connects the APM spheres at some points to the wall of the pipe. The rearrangement of APM elements needs to overcome this adhesive force between the spheres. The reason is that each APM element has a fixed position, due to adhesion and needs to be rearranged. Under compressive load, the epoxy resin and the adjacent area of APM small spheres first undergo a quasielastic deformation. Then, the internal pore structures of a few APM small spheres may show plastic deformation. With further increase in pressure, however, the elastic deformation of the epoxy joint and the APM pipe reach their limits. Gradually increasing the load can finally overcome the friction between the pipe wall and the APM core. Hence, the bonding point is peeled off and the resultant load drops, which are associated with load fluctuations during the repeated yield of the pipe, as reflected in the load-strain curve. It has been demonstrated that the use of a binding filler can increase the interaction between the wall and the filler, thus increasing the overall energy absorption level [10]. There are two reasons for using epoxy foam-bonding filled pipes to improve energy absorption: (1) due to the bonding effect of epoxy resin foaming, a network between APM spheres and the pipe can be formed, which increases the transfer of load from the pipe wall to the inner core. (2) The tearing of the bonded site is caused by the formation of folds on the outer side of the pipe. After the epoxy resin is foamed, the load-transfer mode between the APM elements, or the APM elements and the pipe is not the point of contact, but the network of foaming epoxy resin. Compared with direct bonding, the epoxy foaming process enhances the load transfer, transferring from the point-contact load-transfer network to the surfacecontact load-transfer network [17], and it also enhances the frictional effect with the pipe wall. Such enhancements intensify the interaction between the pipe and the core, thereby widening the region of higher absorption efficiency.

It is evident that the epoxy foam-bonding APM composite pipe is superior to the thin-walled hollow pipe in terms of AE or I. Hence, a combination of foaming and bonding can be used as a new filling process for APM fillers.

\section{Conclusions}

(1) The thin-walled steel pipe undergoes an obvious asymmetric deformation mode and the folds are mainly diamond-shaped. The epoxy-bonding APMfilled pipe deforms in the hybrid form, and the folds are both diamond-shaped and hexagonal. The epoxy foam-bonding APM composite pipe undergoes a typical symmetric deformation mode, and all the formed folds are hexagonal. The latter two form more folds than the thin-walled empty pipe.

(2) Epoxy-bonding APM-filled pipe and epoxy foambonding APM composite pipe enhance the level of compressive load. The epoxy foam-bonding APM composite pipe has the highest $\mathrm{AE}, 3.1$ times more than that of the empty pipe. This was followed by the epoxy-bonding APM composite pipe, whose AE value was 2.63 times that of the empty tube. The epoxy foam-bonding APM composite pipe enters the high-efficiency energy absorption region with $10 \%$ strain and remains till 55\% strain is reached. Compared with the epoxy-bonding APM-filled pipe, the epoxy foam-bonding APM composite pipe further broadens the high-efficiency energy absorption region (the ideal energy absorption efficiency is $70 \%$ or more) and increases the strain range from $30 \%$ $50 \%$ to $20 \% \sim 50 \%$. The ideal energy absorption efficiency of the epoxy foam-bonding APM composite pipe is about $154 \sim 182 \%$ that of the thin-walled empty pipe and $154 \sim 167 \%$ that of the epoxy-bonding APM composite pipe. The foaming process for epoxy resin further enhances the energy absorption performance and can be used as a new filling and bonding process for APM fillers.

\section{Data Availability}

The data used to support the findings of this study are included within the article.

\section{Conflicts of Interest}

The authors declare that there are no conflicts of interest regarding the publication of this paper.

\section{Authors' Contributions}

Yanli Wang and Qiaoyu Guo contributed equally to this work.

\section{References}

[1] J. Baumeister, J. Weise, E. Hirtz, K. Höhne, and J. Hohe, "Applications of aluminum hybrid foam sandwiches in battery housings for electric vehicles," Procedia Materials Science, vol. 4, pp. 317-321, 2014.

[2] K. Stöbener, J. Baumeister, G. Rausch, and M. Rausch, "Forming metal foams by simpler methods for cheaper solutions," Metal Powder Report, vol. 60, no. 1, pp. 12-16, 2005.

[3] O. Friedl, C. Motz, H. Peterlik, S. Puchegger, N. Reger, and R. Pippan, "Experimental investigation of mechanical properties of metallic hollow sphere structures," Metallurgical and Materials Transactions B, vol. 39, no. 1, pp. 135-146, 2008.

[4] X. Luo, J.-Y. Xu, L. Nie, Y. Gao, J. Zhu, and W. Li, "The mechanical behavior of thin-walled tube filled with hollow metal spheres," Composite Structures, vol. 133, pp. 124-130, 2015.

[5] K. Stöbener, D. Lehmhus, M. Avalle, L. Peroni, and M. Busse, "Aluminum foam-polymer hybrid structures (APM aluminum foam) in compression testing," International Journal of Solids and Structures, vol. 45, no. 21, pp. 5627-5641, 2008.

[6] K. Stöbener and G. Rausch, "Aluminium foam-polymer composites: processing and characteristics," Journal of $\mathrm{Ma}$ terials Science, vol. 44, no. 6, pp. 1506-1511, 2009.

[7] J. Hohe, V. Hardenacke, V. Fascio et al., "Numerical and experimental design of graded cellular sandwich cores for multi-functional aerospace applications," Materials \& Design, vol. 39, pp. 20-32, 2012.

[8] M. Vesenjak, M. Borovinšek, T. Fiedler, Y. Higa, and Z. Ren, "Structural characterisation of advanced pore morphology 
(APM) foam elements," Materials Letters, vol. 110, pp. 201203, 2013.

[9] T. Fiedler, M. A. Sulong, M. Vesenjak et al., "Determination of the thermal conductivity of periodic APM foam models," International Journal of Heat and Mass Transfer, vol. 73, pp. 826-833, 2014.

[10] M. A. Sulong, M. Vesenjak, I. V. Belova, G. E. Murch, and T. Fiedler, "Compressive properties of advanced pore morphology (APM) foam elements," Materials Science and Engineering: $A$, vol. 607, pp. 498-504, 2014.

[11] M. Ulbin, M. Borovinšek, Y. Higa, K. Shimojima, M. Vesenjak, and Z. Ren, "Internal structure characterization of AlSi7 and AlSi10 advanced pore morphology (APM) foam elements," Materials Letters, vol. 136, pp. 416-419, 2014.

[12] A. Kovačič and Z. Ren, "On the porosity of advanced pore morphology structures," Composite Structures, vol. 158, pp. 235-244, 2016.

[13] S. Yiatros, O. Marangos, R. A. Votsis, and F. P. Brennan, "Compressive properties of granular foams of adhesively bonded steel hollow sphere blocks," Mechanics Research Communications, vol. 94, pp. 13-20, 2018.

[14] M. Palermo, I. Ricci, S. Silvestri et al., "Preliminary interpretation of shaking-table response of a full-scale 3-storey building composed of thin reinforced concrete sandwich walls," Engineering Structures, vol. 76, pp. 75-89, 2014.

[15] Y. Wang, L. Wang, H. Xu et al., "Preparation and compressive properties of advanced pore morphology (APM) foam elements," in Physics and Engineering of Metallic Materials, pp. 383-390, Springer Proceedings in Physics, Berlin, Germany, 2019.

[16] L. P. Mikkelsen, "A numerical axisymmetric collapse analysis of viscoplastic cylindrical shells under axial compression," International Journal of Solids and Structures, vol. 36, no. 5, pp. 643-668, 1999.

[17] I. Duarte, M. Vesenjak, L. Krstulović-Opara, and Z. Ren, "Compressive performance evaluation of APM (Advanced Pore Morphology) foam filled tubes," Composite Structures, vol. 134, pp. 409-420, 2015.

[18] J. Baumeister, M. Monno, M. Goletti, V. Mussi, and J. Weise, "Dynamic behavior of hybrid APM (advanced pore morphoslogy foam) and aluminum foam filled structures," Metals, vol. 2, no. 2, pp. 211-218, 2012. 


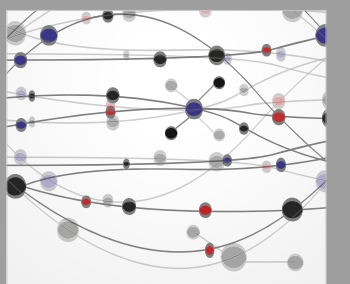

The Scientific World Journal
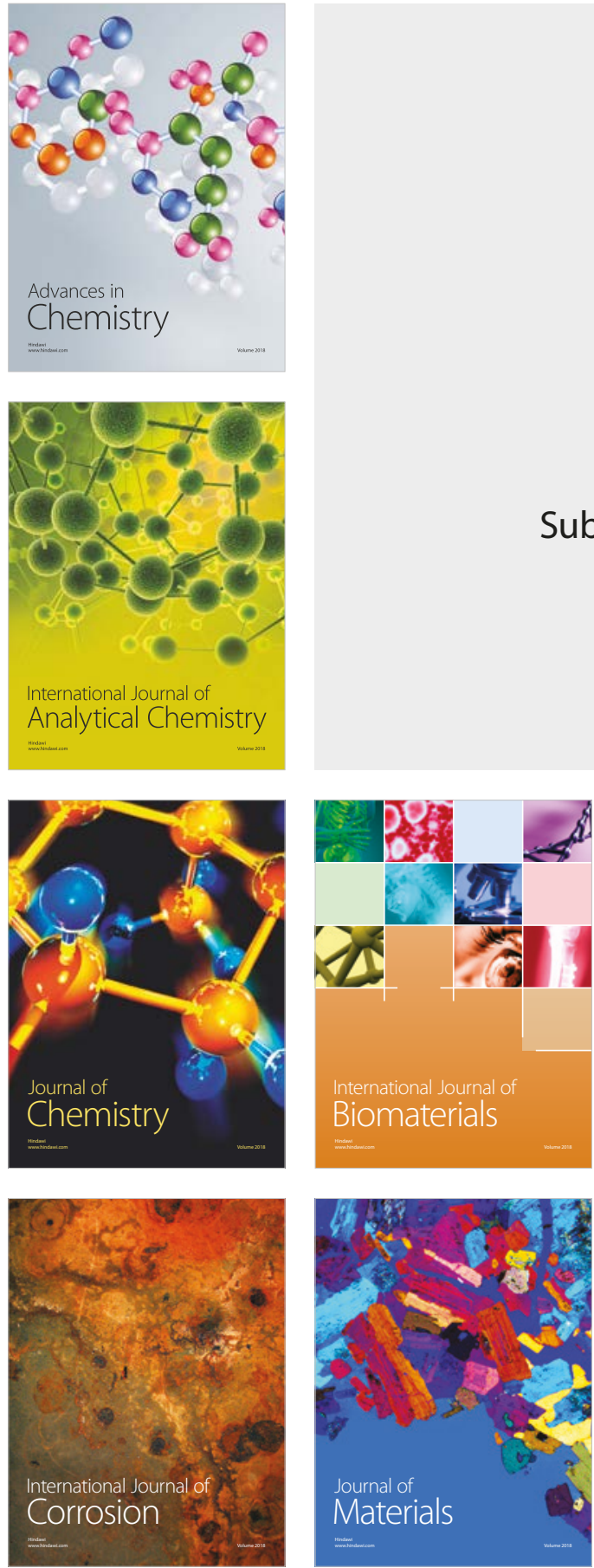

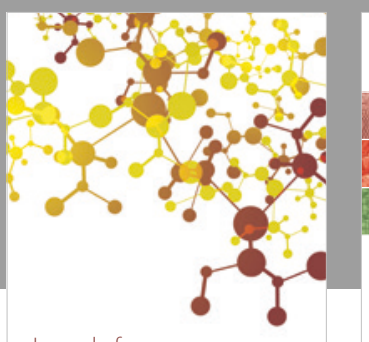

Journal of

Applied Chemistry
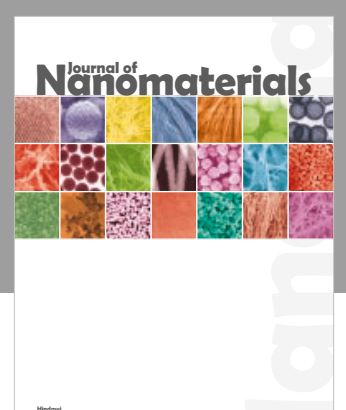

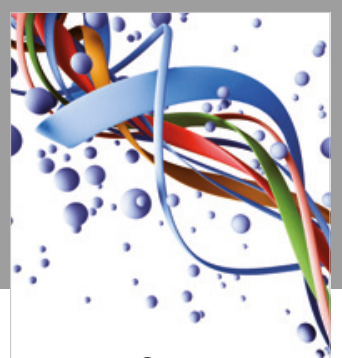

Scientifica

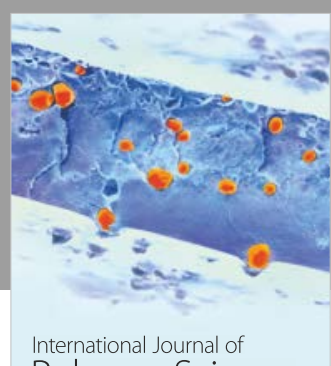

Polymer Science

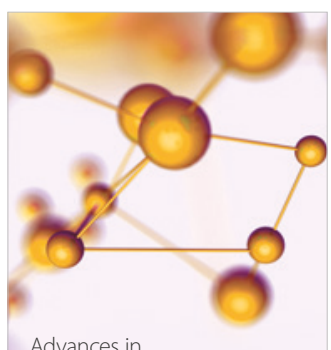

Physical Chemistry
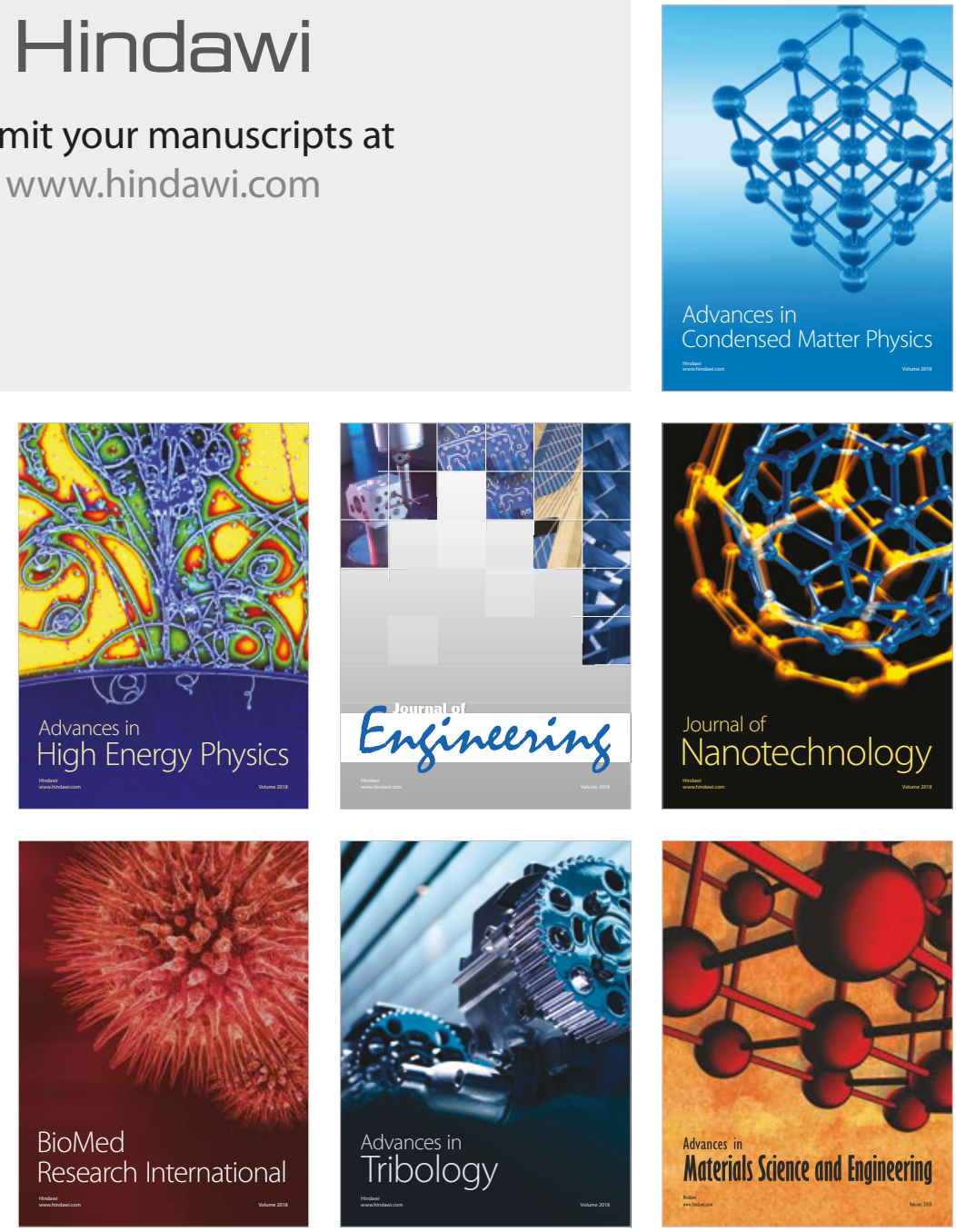